\title{
Perfeccionismo socialmente prescrito y afectividad en población infantil española
}

\author{
María Vicent ${ }^{1}$, Cándido J. Inglés ${ }^{2}$, Ricardo Sanmartín ${ }^{1}$, Carolina Gonzálvez ${ }^{1}$, \\ Lucía Granados Alós ${ }^{3}$ y José Manuel García-Fernández ${ }^{1}$ \\ ${ }^{1}$ Universidad de Alicante (España); ${ }^{2}$ Universidad Miguel Hernández (España); \\ ${ }^{3}$ Universidad Internacional de Valencia (España)
}

El presente estudio tiene por objeto analizar la relación entre el Perfeccionismo Socialmente Prescrito (PSP) y el afecto durante la infancia. Se empleó una muestra compuesta por 804 alumnos de Educación Primaria entre 8 y 11 años $(M=9.57 ; D E=1.12)$, así como la subescala PSP de la Child and Adolescent Perfectionism Scale y la 10-Item Positive and Negative Affect Schedule for Children que evalúa el Afecto Positivo (AP) y Negativo (AN). Se compararon las puntuaciones medias en AP y AN entre los alumnos con altos y bajos niveles de PSP. Los alumnos con alto PSP puntuaron significativamente más alto que sus iguales con bajo PSP tanto en AP como en $\mathrm{AN}$, siendo pequeña la magnitud de las diferencias encontradas. En consonancia, los resultados de las regresiones logísticas revelaron que ambas dimensiones afectivas predijeron positiva y significativamente altas puntuaciones en PSP, con valores de Odd Ratio de 1.08 y 1.05 , respectivamente, para el AP y el AN. Los resultados no coinciden con las evidencias empíricas previas en población adulta y adolescente, lo cual implica que considerar el entorno demandante de perfeccionismo no posee las mismas consecuencias durante la infancia que en edades posteriores.

Palabras clave: Perfeccionismo socialmente prescrito; afecto positivo; afecto negativo; infancia; Educación Primaria.

Socially prescribed perfectionism and affectivity in Spanish child population. This study aims to analyze the relationship between Socially Prescribed Perfectionism (SPP) and affect during childhood. A sample of 804 students aged between 8 and 11 years old $(M=9.57 ; S D=1.12)$, as well as the SPP subscale of the Child and Adolescent Perfectionism Scale and the 10-Item Positive and Negative Affect Schedule for Children, which assess the Positive Affect (PA) and Negative Affect (NA), were employed. The mean scores in PA and NA between students with high and low levels of SPP were compared. Students with high SPP significantly scored higher than their peers with low SPP, both in PA and NA. The effect magnitude of the found differences was small. Accordingly, the results of the logistic regression analysis revealed that both affective dimensions predicted positively and significantly high scores in SPP with Odd Ratio values of 1.08 and 1.05 , respectively, for PA and NA. The results do not match the previous empirical evidence in adult and adolescent population. This implies that the consequences of considering the environment as demanding of perfectionism does not have the same consequences in childhood than in later ages.

Keywords: Socially prescribed perfectionism; positive affect; negative affect; childhood; Primary Education.

Correspondencia: María Vicent. Departamento de Psicología Evolutiva y Didáctica, Universidad de Alicante. Campus de Sant Vicent del Raspeig, Ap. 99. C.P.: E-03080. Alicante (España). E-mail: maria.vicent@ua.es 
El Perfeccionismo Socialmente Prescrito (PSP) engloba la creencia de que las personas que nos rodean esperan que nuestro desempeño sea perfecto, siendo altamente duras y críticas con los errores cometidos (Hewitt y Flett, 2004). Esta faceta interpersonal se considera una de las formas desadaptativas del perfeccionismo multidimensional (e.g., Frost, Heimberg, Holt, Mattia, y Nuebauer, 1993; Gaudreau y Thompson, 2010; Stoeber y Otto, 2006), dada su estrecha relación con una amplia gama de psicopatologías. Sin embargo, poco se sabe acerca de la forma en que las dimensiones perfeccionistas se manifiestan durante la infancia (Morris y Lomax, 2014). De hecho, estudios recientes en población infantil española aseveran que es posible que, en edades tempranas, el PSP no sea tan perjudicial como ha demostrado ser en edades posteriores (Vicent, Inglés, Gonzálvez, Sanmartín, y García-Fernández, 2016).

\section{PSP, afecto positivo y afecto negativo}

El Afecto Positivo (AP; i.e., dimensión de emocionalidad positiva, energética, de afiliación y de dominio de un individuo) y Negativo (AN; dimensión de emocionalidad negativa, caracterizada por estados de ánimo como la aversión, ira, disgusto, nerviosismo, etc.) se consideran indicadores de distintas psicopatologías (e.g., Anderson y Hope, 2008). En lo que respecta al perfeccionismo, existen diversas evidencias empíricas acerca de la forma en que el PSP se vincula con el AP y el AN (Downey y Chang, 2007; Downey, Reinking, Gibson, Cloud, y Chang, 2014; Dunkley, Zuroff, y Blankstein, 2006; Flett, Blankstein, y Hewitt, 2009; Frost et al., 1993; Gaudreau y Verner-Filion, 2012; Ho, Appleton, Cumming, y Duda, 2015; Molnar, Reker, Culp, Sadava, y DeCourville, 2006; Nejad, Ali-Besharat, Haddadi, y Abdolmanafi, 2011; Short y Mazmanian, 2013; Stoeber y Corr, 2015, 2016).

Uno de los primeros trabajos en este sentido fue el desarrollado por Frost et al. (1993), con 553 estudiantes universitarios estadounidenses, concluyendo que el PSP se asocia positiva y significativamente con el AN pero no con el AP. Posteriormente, en un estudio sobre perfeccionismo y salud física con 492 norteamericanos adultos entre 24 y 35 años, Molnar et al. (2006) hallaron que el PSP correlacionó ligeramente, de forma significativa y negativa con el AP y moderada y positivamente con el AN. Además, mediante un sistema de ecuaciones estructurales en el que la relación entre el PSP y la salud física estaba parcialmente mediada por el afecto, se encontró que en dicho modelo, el PSP se asoció con los niveles más bajos de AP y con un mayor AN, lo cual, a su vez, se relaciona con una baja salud física.

Por su parte, Dunkley et al. (2006), en una muestra de 163 estudiantes universitarios norteamericanos con una edad media de 20.02 años $(D E=2.28)$, con medidas retrospectivas y diarias, constataron correlaciones significativas y positivas entre el PSP y el AN y en sentido negativo con el AP, tanto en las medidas retrospectivas como en las diarias. Similarmente, en una muestra de 307 universitarias norteamericanas 
entre 18 y 38 años, Downey y Chang (2007) hallaron que el AN correlacionó positiva y significativamente con el PSP, con magnitudes del efecto moderadas. Asimismo, con una muestra de 92 estudiantes universitarias canadienses con una media de edad de 22.2 años $(D E=6)$, Flett et al. (2009) hallaron que el PSP se asoció significativa y positivamente con el AN y de forma negativa con el AP.

Nejad et al. (2011) evaluaron el efecto del AP y AN en la relación entre el perfeccionismo y la salud física en 234 adultos iraníes entre 24 y 49 años. En el análisis de correlaciones, se observó que el PSP correlacionó muy ligeramente pero de forma positiva y significativa con el Afecto Positivo. Empleando una muestra de 208 atletas canadienses entre 14 y 18 años, Gaudreau y Verner-Filion (2012) pusieron a prueba el modelo 2x2 del perfeccionismo con las dimensiones de PSP y Perfeccionismo AutoOrientado (PAO; i.e., tendencia a autoimponerse normas de desempeño excesivamente altas y la motivación por alcanzar la perfección). Los autores encontraron que el PSP Puro (altos niveles de PSP y bajos niveles de PAO) se asoció con menos niveles de AP que cualquier otra combinación de PAO y PSP.

Short y Mazmanian (2013) hallaron, con una muestra compuesta por 213 universitarios canadienses entre 18 y 28 años, correlaciones significativas y positivas entre el PSP y el AN, así como en sentido negativo con el AP. Posteriormente, Downey et al. (2014), en una muestra de 480 universitarios estadounidenses entre 17 y 61 años, encontraron correlaciones positivas y significativas entre el PSP y el AN, tanto en varones como en mujeres, así como en sentido negativo con el AP, únicamente en la muestra femenina.

Más recientemente, Ho et al. (2015) compararon las puntuaciones entre 205 atletas ingleses oyentes $\left(M_{\text {edad }}=18.8\right.$ años; $\left.D E=3.9\right)$ y 212 con discapacidad auditiva $\left(M_{\text {edad }}=27.3\right.$ años; $\left.D E=9.3\right)$. El análisis correlacional evidenció que, en la muestra de oyentes, el PSP y el AN se asociaron positiva y significativamente. No se observaron correlaciones significativas para la muestra de atletas con discapacidad auditiva. Por último, Stoeber y Corr (2015, 2016) encontraron, en 388 universitarios ingleses $\left(M_{\text {edad }}=19.8\right.$ años; $\left.D E=4.0\right)$, que el PSP correlacionó significativamente y en sentido positivo con el AN. Contrariamente, el PSP se asoció de forma negativa con el AP. Los resultados de las regresiones múltiples manifestaron que el PSP tiene un efecto negativo y significativo sobre el AP, así como positivo sobre el AN, aun añadiendo el resto de variables analizadas en su estudio (Stoeber y Corr, 2015).

\section{El presente estudio}

De la revisión de la literatura científica que ha examinado la relación entre el afecto y el PSP, se desprenden las siguientes conclusiones. En primer lugar, se observa una tendencia a emplear población de habla inglesa, ya sea norteamericana o bien británica, encontrando como única excepción el estudio de Nejad et al. (2011), con 
población iraní. Asimismo, se observa un predominio de investigaciones con estudiantes universitarios (Downey y Chang, 2007; Downey et al., 2014; Dunkley et al., 2006; Flett et al., 2009; Frost et al., 1993; Short y Mazmanian, 2013; Stoeber y Corr, 2015, 2016) o población general adulta (Molnar et al., 2006; Nejad et al., 2011). Son excepciones de esta tendencia los estudios de Ho et al. (2015), elaborado con atletas adultos, y Gaudreau y Verner-Filion (2012), también con atletas, pero en edad adolescente. Por tanto, se desconocen evidencias empíricas previas sobre la relación entre el afecto y el PSP en población infantil.

En cuanto a la metodología de los estudios, en general, los datos que ofrecen los trabajos revisados son de carácter correlacional, así como resultados derivados de análisis de regresión múltiple (Nejad et al., 2011; Stoeber y Corr, 2015), regresión jerárquica (Dunkley et al., 2006) y ecuaciones estructurales (Molnar et al., 2006).

El presente trabajo pretende solventar las limitaciones detectadas examinando la relación entre el PSP y el afecto en una muestra representativa de población infantil española. Este objetivo general se concreta en: (a) analizar si existen diferencias en las puntuaciones medias en AP y AN, entre los niños con altos y bajos niveles de PSP y (b) examinar la capacidad predictiva del AP y AN sobre las altas puntuaciones en PSP. Así, teniendo en consideración que la literatura científica ha encontrado generalmente que el PSP se asocia de forma significativamente negativa con el AP (Dunkley et al., 2006; Flett et al., 2009; Ho et al., 2015; Molnar et al., 2006; Short y Mazmanian, 2013; Stoeber y Corr, 2015, 2016), se espera que los alumnos con alto PSP puntúen significativamente más bajo en AP que sus iguales con bajo PSP, así como que el AP sea un predictor significativo y negativo de altos niveles en PSP (Hipótesis 1). Por otro lado, en consonancia con los estudios previos que han encontrado que el PSP se asocia significativamente y de forma positiva con el AN (Downey y Chang, 2007; Downey et al., 2014; Dunkley et al., 2006; Flett et al., 2009; Frost et al., 1993; Ho et al., 2015; Molnar et al., 2006; Short y Mazmanian, 2013; Stoeber y Corr, 2015, 2016), se espera que los alumnos con alto PSP puntúen significativamente más alto en AN que sus iguales con bajo PSP, así como que el AN sea un predictor significativo y positivo de altos niveles en PSP (Hipótesis 2).

\section{MÉTODO}

\section{Participantes}

La muestra de este estudio se seleccionó a través de un muestreo aleatorio por conglomerados (zonas geográficas de la provincia de Alicante, Murcia y Albacete: centro, norte, sur, este y oeste). Siguiendo este procedimiento se seleccionaron un total de 10 centros públicos de Educación Primaria, de los cuales se escogió aleatoriamente un grupo por cada curso académico desde $3^{\circ}$ hasta $6^{\circ}$ curso. De este forma, se reclutó una 
muestra inicial de 982 estudiantes, de los cuales se excluyeron un $4.38 \%$ por errores u omisiones en las respuestas, un $3.90 \%$ por tratarse de alumnado repetidor, un $4.89 \%$ por no presentar el nivel mínimo de lectura requerido y un $4.96 \%$ por no contar con el consentimiento parental. Así, la muestra definitiva se compuso de 804 alumnos entre 8 y 11 años $\left(M_{\text {edad }}=9.57 ; D E=1.12\right)$. La prueba $\chi^{2}$ de la homogeneidad de la distribución de frecuencias $\left(\chi^{2}=5.08 ; p=.17\right)$ reveló la ausencia de diferencias estadísticamente significativas para los ocho grupos de sexo por edad (véase tabla 1).

Tabla 1. Número (porcentaje) de participantes de la muestra total clasificados en función del sexo y la edad

\begin{tabular}{cccccc}
\hline & 8 años & 9 años & 10 años & 11 años & Total \\
\hline \multirow{2}{*}{ Varones } & 88 & 100 & 90 & 138 & 416 \\
& $(10.9 \%)$ & $(12.4 \%)$ & $(11.2 \%)$ & $(17.2 \%)$ & $(51.7 \%)$ \\
\hline \multirow{2}{*}{ Mujeres } & 88 & 118 & 92 & 90 & 388 \\
& $(10.9 \%)$ & $(14.7 \%)$ & $(11.4 \%)$ & $(11.2 \%)$ & $(48.3 \%)$ \\
\hline \multirow{2}{*}{ Total } & 176 & 218 & 182 & 228 & 804 \\
& $(21.9 \%)$ & $(27.1 \%)$ & $(22.6 \%)$ & $(28.4 \%)$ & $(100 \%)$ \\
\hline
\end{tabular}

\section{Instrumentos}

The Child and Adolescent Perfectionism Scale (CAPS; Flett, Hewitt, Boucher, Davidson, y Munro, 2000; García-Fernández, Inglés, y Vicent, 2016).

Se empleó la versión española de la subescala PSP de la CAPS, validada en población infantil por el propio equipo de investigación. La CAPS es el instrumento de perfeccionismo infantil más empleado a nivel internacional para evaluar dicho constructo en población infantojuvenil a partir de 8 años de edad (García-Fernández et al., 2016) y tiene como finalidad evaluar la tendencia a considerar el entorno como altamente demandante de perfeccionismo (e.g., "Mi familia espera que yo sea perfecto"). En su validación española (García-Fernández, Inglés, y Vicent, 2016), el PSP únicamente mantuvo 5 de los 10 ítems que componen su estructura original (Flett et al., 2000), valorados a través de una escala tipo Likert de 5 puntos, con niveles de consistencia interna y estabilidad temporal aceptables $\left(\alpha=.75\right.$ y $\left.r_{x x}=.74\right)$. Asimismo, estudios previos con población infantil española ofrecen resultados acerca de su asociación con los cinco grandes rasgos de la personalidad, la ansiedad escolar y la conducta agresiva (Inglés, García-Fernández, Vicent, Gonzálvez, y Sanmartín, 2016; Vicent et al., 2016)

The 10-Item Positive and Negative Affect Schedule for Children (10-item PANAS-C; Ebesutani et al., 2012).

El 10-item PANAS-C es la versión reducida del Positive and Negative Affect Schedule for Children (PANAS-C; Laurent et al., 1999) que evalúa el afecto en niños de 6 a 18 años. El cuestionario se compone de dos subescalas denominadas AP y AN con cinco ítems cada una ("alegre, animado, feliz, con energía, orgulloso" y "deprimido, enfadado, temeroso/con miedo, asustado y triste", respectivamente) valorados a través de una escala de respuesta tipo Likert de 5 puntos. El instrumento cuenta con una versión 
para niños y otra para padres, no obstante, en nuestro estudio únicamente se empleó la versión para niños. En su validación inicial, se obtuvieron coeficientes de fiabilidad, alfa de Cronbach de .86 para el AP y .82 para AN (Ebesutani et al., 2012).

Los coeficientes de fiabilidad, alfa de Cronbach, para este estudio fueron de .75 y .76 para el AP y AN, respectivamente.

\section{Procedimiento}

Se celebró una reunión con los responsables de los centros educativos para informarles sobre nuestros objetivos y solicitar su colaboración. Seguidamente, se solicitó el consentimiento informado por escrito de los padres o tutores legales de los participantes seleccionados. Una vez obtenido dicho justificante se procedió a aplicar las pruebas en una única sesión de 30 minutos, en horario escolar, de forma grupal y estando presente un investigador en todo momento. Al inicio de la sesión, el investigador hizo hincapié en el carácter voluntario y anónimo de la actividad. Los participantes fueron tratados en todo momento bajo los criterios éticos que rigen la investigación científica.

\section{Análisis de datos}

En primer lugar, se dicotomizó la variable PSP en altas ( $\geq$ al centil 75) y bajas $(\leq$ al centil 25) puntuaciones. Posteriormente, se empleó la prueba $t$ de Student de diferencias de medias para medidas independientes, con el fin de hallar las posibles diferencias entre los estudiantes con alto y bajo PSP en las puntuaciones medias reportadas para AP y AN. Asimismo, se calculó la magnitud de las diferencias halladas a través del índice $d$ de Cohen (diferencia de media tipificada), el cual se interpreta de la siguiente manera: pequeño (valores entre .20 y .50), moderado (valores entre .51 y .79) y grande (valores iguales o superiores a .80).

En segundo lugar, se empleó el método de regresión logística binaria siguiendo el procedimiento de regresión por pasos hacia delante, basado en el estadístico de Wald, para analizar la capacidad predictiva del AP y AN sobre las altas puntuaciones en PSP. Esta capacidad de predicción es estimada por el estadístico Odd Ratio $(O R)$ que se interpreta de la siguiente manera: $O R>1$ indica predicción en sentido positivo, $O R<1$ indica predicción en sentido negativo, mientras que el valor 1 indica que no hay predicción (De Maris, 2003).

Todos los análisis fueron realizados con el programa informático SPSS versión 22.0 .

\section{RESULTADOS}

En la tabla 2 se ofrecen los resultados para las diferencias en las puntuaciones medias en afecto entre los grupos con alto y bajo PSP. Tal y como puede observarse, los 
alumnos con alto PSP puntuaron significativamente más alto que sus iguales con bajo PSP, tanto en AP como en AN, con tamaños del efecto pequeños para ambos casos ( $d=.30$ y .17 , respectivamente).

Tabla 2. Medias, desviaciones típicas y tamaño del efecto para el Afecto Positivo y Negativo obtenidas por los grupos alto PSP y bajo PSP

\begin{tabular}{lcccccccccc}
\hline & Prueba Levene & Grupo Alto PSP & Grupo Bajo PSP & \multicolumn{3}{c}{$\begin{array}{c}\text { Sig. Estadística y } \\
\text { magnitud de diferencias }\end{array}$} \\
\hline $\begin{array}{l}\text { Dimensiones del } \\
\text { 10-item PANAS-C }\end{array}$ & $F$ & $p$ & $M$ & $D E$ & $M$ & $D E$ & $t 401$ & $p$ & $d$ \\
\hline Afecto Positivo & .02 & .874 & 20.29 & 4.13 & 19.03 & 4.14 & -4.05 & $<.001$ & .30 \\
\hline Afecto Negativo & 5.69 & .017 & 8.73 & 3.98 & 8.07 & 3.49 & -2.34 & .020 & .17 \\
\hline
\end{tabular}

Tabla 3. Regresión logística binaria para la probabilidad de presentar alto PSP en función de las dos subescalas del 10-item PANAS-C

\begin{tabular}{lccccccccc}
\hline Variable & & $\chi^{2}$ & $R^{2}$ & B & ET & Wald & $p$ & OR & I.C. 95\% \\
\hline $\begin{array}{l}\text { Afecto } \\
\text { Positivo }\end{array}$ & $\begin{array}{c}\text { Clasificados } \\
\text { corec.: 59.1\% }\end{array}$ & 16.11 & .03 & .07 & .02 & 15.76 & $<.001$ & 1.08 & $1.04-1.12$ \\
\cline { 2 - 10 } & Constante & -1.10 & .37 & 8.95 & .003 & .33 & \\
\hline $\begin{array}{l}\text { Afecto } \\
\text { Negativo }\end{array}$ & $\begin{array}{c}\text { Clasificados } \\
\text { correc.: } 58.2 \%\end{array}$ & 5.36 & .01 & .05 & .02 & 5.15 & .02 & 1.05 & $1.01-1.09$ \\
\cline { 2 - 9 } & Constante & -.06 & .19 & .12 & .73 & .94 & \\
\hline
\end{tabular}

Nota: Clasificados correc. $=$ Clasificados correctamente.

Los resultados para las regresiones logísticas binarias que analizan la capacidad predictiva de las dos dimensiones afectivas sobre el alto PSP pueden observarse en la tabla 3 . La proporción de casos correctamente clasificados fue del $58.2 \%$ y el $59.1 \%$ y la $R^{2}$ de Nahelkerke de .01 y .03 , respectivamente, para la capacidad predictiva del AN y AP, respectivamente. Se obtuvieron niveles de $O R$ de 1.05 y 1.08 , indicando que la probabilidad de presentar altas puntuaciones en PSP aumenta un 5\% y un $8 \%$ por cada punto que se incrementan los niveles en AN y AP, respectivamente.

\section{DISCUSIÓN Y CONCLUSIONES}

El objetivo del presente estudio consistió en analizar la relación entre el PSP y las dos dimensiones de la afectividad. Los resultados mostraron que los alumnos con altos niveles en dicha dimensión obtuvieron significativamente puntuaciones más altas tanto en AP como AN que sus iguales con bajo PSP. En la misma línea, se halló que ambas dimensiones del afecto predijeron positivamente y de forma significativa altos niveles de PSP. Estos resultados sustentan la Hipótesis 1 del presente estudio, así como los resultados de los trabajos previos que han encontrado que el PSP se asocia significativamente y de forma positiva con el AN (Downey y Chang, 2007; Downey et al., 2014; Dunkley et al., 2006; Flett et al., 2009; Frost et al., 1993; Ho et al., 2015; Molnar et al., 2006; Short y Mazmanian, 2013; Stoeber y Corr, 2015, 2016). Ciertamente, el PSP ha sido asociado con la tendencia a experimentar pensamientos 
negativos repetitivos (Randles, Flett, Nash, McGregor, y Hewitt, 2010), así como sentimientos de culpa y vergüenza, especialmente en situaciones de fracaso (Stoeber, Kempe, y Keogh, 2008), posiblemente derivados del miedo y la preocupación por ser criticados o castigados por ello, o bien por perder la aprobación y la estima de los demás, al no poder satisfacer sus expectativas. También cabe considerar que se ha sugerido incluso que la relación entre el PSP y el AN podría estar mediada por la preocupación y la rumia psicológica (e.g., Short y Mazmanian, 2013). De cualquier forma, se trata de un rasgo de la personalidad propenso a la afectividad negativa.

Sin embargo, la asociación positiva hallada entre el PSP y el AP contradice la Hipótesis 2 de este estudio, según la cual se esperaba que los alumnos con alto PSP puntuaran significativamente más bajo en AP que sus iguales con bajo PSP, así como que el AP resultase un predictor significativo y negativo de altos niveles en PSP, en concordancia con la literatura previa al respecto (Dunkley et al., 2006; Flett et al., 2009; Ho et al., 2015; Molnar et al., 2006; Short y Mazmanian, 2013; Stoeber y Corr, 2015, 2016). Cabe mencionar, que existen investigaciones que suponen una excepción a estos resultados habituales en la literatura científica. Así, Nejad et al. (2010) hallaron que la relación entre el PSP y el AP fue en sentido positivo, mientras que Frost et al. (1993) encontraron que esta relación no alcanzó la significación estadística.

Estas divergencias entre las conclusiones de los estudios previos y el presente trabajo pueden deberse a aspectos culturales o bien a la edad de la muestra, ya que se desconocen investigaciones anteriores que hayan examinado la relación entre el PSP y el afecto en población infantil y española, así como a los instrumentos empleados para la evaluación de las variables. Igualmente, cabe mencionar que este estudio se ha centrado en población con altos niveles (superiores al centil 75) en PSP y, por tanto, los resultados podrían variar ligeramente con respecto a aquellos que examinaron la relación entre el afecto y el PSP, sin atender específicamente a población con elevadas puntuaciones en esta última dimensión. Pese a ello, la explicación acerca de la relación positiva entre el PSP y el AP no está clara.

Una posible justificación sería que, durante la infancia, la demanda de perfeccionismo por parte de los demás sea interpretada como un signo de preocupación y creencia en sus habilidades y su capacidad, aumentando de esta forma la propia autoestima, autoeficacia y la participación placentera en el entorno. Asimismo, otra posibilidad es que el PSP se asocie con diferentes consecuencias emocionales en función de las interacciones con otras variables, como, por ejemplo, el éxito o el fracaso en el desempeño, o los conflictos diarios. Posiblemente, durante estas primeras etapas de la vida, los estándares impuestos sean más fácilmente alcanzables por los niños perfeccionistas, recibiendo por ello recompensas, elogios y premios de sus familiares o profesores y, por tanto, aumentando los sentimientos positivos, como el orgullo o la felicidad (e.g., Ramos-Díaz, Rodríguez-Fernández, Revuelta, y Axpe, 2016). Al mismo 
tiempo, probablemente las emociones positivas coexistan con el miedo y la preocupación por el hecho de no poder alcanzar las expectativas del entorno, así como con la infelicidad, culpa y disgusto provocado por las críticas recibidas tras los fracasos. De hecho, conviene recordar que la investigación ha apoyado tradicionalmente la idea de la supuesta independencia entre el AP y AN (Egloff, 1998; Inglés, Gonzálvez, García-Fernández, Vicent, y Martínez-Monteagudo, 2016; Tellegen, Watson, y Clark, 1999; Tuccitto, Giaccobi, y Leite, 2010; Watson y Clark, 1997; Watson, Clark, y Tellegen, 1988; Watson y Tellegen, 1985), lo cual significa que no necesariamente ambas dimensiones deben comportarse inversamente. En este sentido, la literatura previa ha identificado diversos perfiles afectivos en función de la combinación entre altos y bajos niveles de AP y AN (e.g., Norlander, Bood, y Archer, 2002), entre los que se encuentra el denominado perfil Afectivo Alto (altas puntuaciones tanto en AP como en AN). Este perfil presenta resultados ambiguos en términos de adaptación y ajuste psicológico. Por ejemplo, los individuos con un perfil Afectivo Alto muestran una elevada persistencia y motivación de logro y son altamente trabajadores. Sin embargo, también suelen ser menos autónomos y más pesimistas, con tendencia a la preocupación y a la rumia sobre sus propias habilidades, así como altamente dependientes de las valoraciones externas, entre otros aspectos (García, Adrianson, Archer, y Rosenberg, 2015). No obstante, no existen evidencias previas acerca de la forma en que dichos perfiles afectivos se manifiestan durante la infancia.

Cabe mencionar que esta investigación tiene diversas limitaciones. En primer lugar, dado su carácter transversal, no es posible establecer relaciones de causalidad entre las variables examinadas. Aspecto que podría examinarse en el futuro a través del método de ecuaciones estructurales o empleando datos longitudinales con el fin de comprobar si efectivamente existe una relación causa-efecto y en tal caso, la dirección de dicha relación. Asimismo, pese a que el tamaño muestral garantiza la representatividad de alumnado español entre 8 y 11 años, no es posible generalizar nuestros resultados a otras edades o poblaciones. Puesto que se ha sugerido que el perfeccionismo podría estar influenciado por la cultura de pertenencia (Marten-Dibartolo y Rendón, 2012), sería de interés que futuros trabajos examinasen la relación entre el PSP y el AP y AN en población infantil perteneciente a otros países, con el fin de averiguar si los resultados obtenidos en este estudio son generalizables a otras culturas, así como emplear población clínica o muestras específicas como por ejemplo participantes con altas capacidades (Ramiro, Guzmán, Menacho, López, y García, 2016). Por otro lado, es necesario recordar que este estudio se asienta bajo la perspectiva del perfeccionismo infantil defendida por Flett et al. (2000). En este sentido, sería conveniente comprobar si los resultados obtenidos en este trabajo son replicables empleando otras medidas de constructos semejantes como la subescala Presión Externa del Inventario de Perfeccionismo Infantil (Lozano-Fernández, García-Cueto, Martín-Vázquez, y 
Lozano-González, 2012). Por último, este trabajo no ha tenido en consideración variables de interés como por ejemplo el sexo. Teniendo en cuenta que estudios previos han encontrado divergencias atribuibles al sexo en el vínculo entre PSP y el afecto (Downey et al., 2014), sería de interés comprobar si la relación hallada entre las variables del presente estudio es consistente cuando se examina en ambos sexos, de forma independiente. Por último, sería de interés contrastar en el futuro la forma en que los perfiles afectivos se asocian con el PSP, y comprobar si, efectivamente, los sujetos con un perfil Afectivo Alto obtienen puntuaciones significativamente más elevadas en PSP que los tres perfiles restantes, así como indagar sobre las consecuencias emocionales que implica dicho perfil durante la infancia.

Pese a las limitaciones, este estudio supone un aporte novedoso para la investigación del perfeccionismo infantil, en tanto que se trata, desde nuestro conocimiento, del primer trabajo que ha examinado la relación entre el PSP y el afecto en población infantil y/o española. Los resultados sugieren que durante la niñez, el PSP se asocia positivamente tanto con el AP como con el AN, al contrario que en etapas más avanzadas, en las que el PSP se vincula positivamente con el AN pero en sentido negativo con el AP. Ello implica que para los niños, la percepción de un entorno demandante de perfeccionismo y altamente crítico no posee las mismas consecuencias que para los adultos, al menos en lo que respecta a la afectividad, lo cual justifica la necesidad de seguir indagando en este tópico durante la infancia, pues los resultados de la investigación en otras poblaciones pueden no ser generalizables a la etapa infantil.

\section{Agradecimientos}

Parte de esta investigación ha sido subvencionada por la ayuda para la contratación de personal investigador en formación predoctoral, Programa VALi+d (ACIF/2014/368), concedida a Maria Vicent, la ayuda para contratos destinados a la formación de doctores-UA FPU 2015-5995, concedida a Ricardo Sanmartín, así como el proyecto "Evaluación de la ansiedad escolar y su relación con variables psicoeducativas en la infancia. Estudio de la eficacia de un programa preventivo" (EDU2012-35124).

\section{REFERENCIAS}

Anderson, E.R. y Hope, D.A. (2008). A review of the tripartite model for understanding he link between anxiety and depression in youth. Clinical Psychology Review, 28, 275-287. doi: 10.1016/j.cpr.2007.05.004

De Maris, A. (2003). Logistic regression. En J.A. Schinka y W.F. Velicer (Eds.), Research methods in psychology (pp. 509-532). Nueva Jersey: John Wiley y Sons.

Downey, C.A. y Chang, E.C. (2007). Perfectionism and symptoms of eating disturbances in female college students: considering the role of negative affect and body dissatisfaction. Eating Behaviors, 8, 497-503. doi: 10.1016/j.eatbeh.2007.02.002 
Downey, C.A., Reinking, K.R., Gibson, J.M., Cloud, J.A., y Chang, E.C. (2014). Perfectionistic cognitions and eating disturbance: distinct mediational models for males and females. Eating Behaviors, 15, 419-426. doi: 10.1016/j.eatbeh.2014.04.020

Dunkley, D.M., Zuroff, D.C., y Blankstein, K.R. (2006). Specific perfectionism components versus self-criticism in predicting maladjustment. Personality and Individual Differences, 40, 665-676. doi: 10.1016/j.paid.2005.08.008

Ebesutani, C., Regan, J., Smith, A., Reise, S., Higa-McMillan, C., y Chorpita, B.F. (2012). The 10Item Positive and Negative Affect Schedule for Children, Child and Parent Shortened Versions: application of item response theory for more efficient assessment. Journal of Psychopathology and Behavioral Assessment, 34(2), 191-203. doi: 10.1007/s10862-0119273-2

Egloff, B. (1998). The independence of positive and negative affect depends on the affect measure. Personality and Individual Differences, 25, 1101-1109. doi: 10.1016/S01918869(98)00105-6

Flett, G.L., Blankstein, K.R., y Hewitt, P.L. (2009). Perfectionism, performance, and state positive affect and negative affect after a classroom test. Canadian Journal of School Psychology, 24, 4-18. doi: 10.1177/0829573509332457

Flett, G.L., Hewitt, P.L., Boucher, D.J., Davidson, L.A., y Munro, Y. (2000). The ChildAdolescent Perfectionism Scale: development, validation, and association with adjustment. Manuscrito inédito, York University, Toronto, Ontario, Canadá.

Frost, R.O., Heimberg, R.G., Holt, C.S., Mattia, J.I., y Nuebauer, M. (1993). A comparison of two measures of perfectionism. Personality and Individual Differences, 14, 119-126. doi: 10.1016/0191-8869(93)90181-2

García, D., Adrianson, L., Archer, T., y Rosenberg, P. (2015). The dark side of the affective profiles: differences and similarities in psychopathy, Machiavellianism, and Narcissism. SAGE Open, 1-14. doi: 10.1177/2158244015615167

García-Fernández, J.M., Inglés, C.J., y Vicent, M. (2016). Escala de perfeccionismo para niños y adolescentes. Manual interno. Universidad de Alicante, España.

García-Fernández, J.M., Inglés, C.J., Vicent, M., Gonzálvez, C., Gómez-Núñez, M.I., y Poveda-Serra, P. (2016). Perfeccionismo durante la infancia y la adolescencia. Análisis bibliométrico y temático (2004-2014). Revista Iberoamericana de Psicología y Salud, 7(2), 79-88. doi: 10.1016/j.rips.2016.02.001

Gaudreau, P. y Thompson, A. (2010). Testing a 2 x 2 model of dispositional perfectionism. Personality and Individual Differences, 48, 532-537. doi: 10.1016/j.paid.2009.11.031

Gaudreau, P. y Verner-Filion, J. (2012). Dispositional perfectionism and well-being: a test of the 2 $\mathrm{x} 2$ model of perfectionism in the sport domain. Sport, Exercise and Performance Psychology, 1(1) 29-43. doi: 10.1037/a0025747

Hewitt, P.L. y Flett, G.L. (2004). Multidimensional Perfectionism Scale (MPS): Technical manual. Toronto: Multi-Health Systems.

Ho, M.S.H., Appleton, P.R., Cumming, J., y Duda, J.L. (2015). Examination the relationship between perfectionism dimensions and burning out symptoms in deaf and hearing athletes. Journal of Clinical Sport Psychology, 9, 156-172. doi: 10.1123/jcsp.2014-0035

Inglés, C.J., García-Fernández, J.M., Vicent, M., Gonzálvez, C., y Sanmartín, R. (2016). Profiles of perfectionism and school anxiety: a review of the $2 \times 2$ model of dispositional perfectionism in child population. Fontiers in Psychology, 7, 1403. doi: 10.3389/fpsyg.2016.01403

Inglés, C.J., Gonzálvez, C., García-Fernández, J.M., Vicent, M., y Martínez-Montegudo, M.C. (2015). Current status of research on school refusal. European Journal of Education and Psychology, 8(1), 37-52. doi: 10.1016/j.ejeps.2015.10.005 
Laurent, J., Catanzaro, S.J., Joiner, T.E., Rudolph, K.D., Potter, K.I., Lambert, S., ... Gathright, T. (1999). A measure of positive and negative affect for children: Scale development and preliminary validation. Psychological Assessment, 11, 326-338. doi: 10.1037/10403590.11.3.326

Lozano-Fernández, L.M., García-Cueto, E., Martín-Vázquez, M., y Lozano-González, L. (2012). Desarrollo y validación del Inventario de Perfeccionismo Infantil (I.P.I.). Psicothema, 24(1), 149-155.

Marten-Dibartolo, P. y Rendón, M.J. (2012). A critical examination of the construct of perfectionism and its relationship to mental health in Asian and African Americans using a cross-cultural framework. Clinical Psychology Review, 32(3), 139-152. doi: 10.1016/j.cpr.2011.09.007

Molnar, D.S., Reker, D.L., Culp, N.A., Sadava, S.W., y DeCourville, N.H. (2006). A mediated model of perfectionism, affect, and physical health. Journal of Research in Personality, 40, 482-500. doi: 10.1016/j.jrp.2005.04.002

Morris, L. y Lomax, C. (2014). Review: assessment, development, and treatment of childhood perfectionism: a systematic review. Child and Adolescent Mental Health, 19(4), 225234. doi:10.1111/camh.12067

Nejad, E.M., Ali-Besharat, M., Haddadi, P., y Abdolmanafi, A. (2011). Mediation effects of positive and negative affect on the relationship between perfectionism and physical health. Procedia-Social and Behavioral Sciences, 30, 176-181. doi: 10.1016/j.sbspro.2011.10.035

Norlander, T., Bood, S.A., y Archer, T. (2002). Performance during stress: Affective personality, age, and regularity of physical exercise. Social Behavior and Personality: an International Journal, 30(5), 495-508. doi: 10.2224/sbp.2002.30.5.495

Ramiro, P., Navarro, J.I., Menacho, I., López, M., y García, M.A. (2016). Bienestar psicológico en personas con alta capacidad intelectual. European Journal of Education and Psychology, 9(2), 72-78. doi: 10.1016/j.ejeps.2015.12.001

Ramos-Díaz, E., Rodríguez-Fernández, A., Revuelta, L., y Axpe, I. (2016). El rol mediador de la implicación escolar sobre el apoyo del profesorado y la satisfacción con la vida. European Journal of Investigation in Health, Psychology and Education, 6(3), 151-165. doi: 10.1989/ejihpe.v6i3.178

Randles, D., Flett, G.L., Nash, K.A., McGregor, I.D., y Hewitt, P.L. (2010). Dimensions of perfectionism, behavioral inhibition, and rumination. Personality and Individual Differences, 49, 83-87. doi: 10.1016/j.paid.2010.03.002

Short, M.M., y Mazmanian, D. (2013). Perfectionism and negative repetitive thoughts: examining a multiple mediator model in relation to mindfulness. Personality ad Individual Differences, 55, 716-721. doi: 10.1016/j.paid.2013.05.026

Stoeber, J. y Corr, P.J. (2015). Perfectionism, personality, and affective experiences: new insights from revised reinforcement sensitivity theory. Personality and Individual Differences, 86, 354-359. doi: 10.1016/j.paid.2015.06.045

Stoeber, J. y Corr, P.J. (2016). A short empirical note on perfectionism and flourishing. Personality and Individual Differences, 90, 50-53. doi: 10.1016/j.paid.2015.10.036.

Stoeber, J., Kempe, T., y Keogh, E.J. (2008). Facets of self-oriented and socially prescribed perfectionism and feelings of pride, shame, and guilt following success and failure. Personality and Individual Differences, 44, 1506-1516. doi: 10.1016/j.paid.2008.01.007

Stoeber, J. y Otto, K. (2006). Positive conceptions of perfectionism: approaches, evidence, challenges. Personality and Social Psychology Review, 10, 295-319. doi: 10.1207/s15327957pspr1004_2 
Tellegen, A., Watson, D., y Clark, L.A. (1999). On the dimensional and hierarchical structure of affect. Psychological Science, 10, 297-303.

Tuccitto, D.E., Giaccobi, P.R., y Leite, W.L. (2010). The internal structure of positive and negative affect: a confirmatory factor analysis of the PANAS. Educational and Psychological Measurement, 70(1), 125-141.

Vicent, M., Inglés, C.J., Gonzálvez, C., Sanmartín, R., y García-Fernández, J.M. (2016). Perfeccionismo socialmente prescrito y los cinco grandes rasgos de la personalidad en niños españoles. European Journal of Investigation in Health Psychology and Education, 6(2), 107-118.

Watson, D. y Clark, L.A. (1997). Measurement and mismeasurement of mood: recurrent and emergent issues. Journal of Personality Assessment, 68, 267-296. doi: 10.1207/s15327752jpa6802_4

Watson, D., Clark, L.A., y Tellegen, A. (1988). Development and validation of brief measures of positive and negative affect: the PANAS scales. Journal of Personality and Social Psychology, 54, 1063-1070.

Watson, D. y Tellegen, A. (1985). Toward a consensual structure of mood. Psychological Bulletin, 98, 219-235.

Recibido: 4 de octubre de 2016 Recepción Modificaciones: 14 de diciembre de 2016 Aceptado: 16 de diciembre de 2016 